Supporting Information for:

\title{
Asymmetric Reduction and Enantioselective Oxidation Using W110A Secondary Alcohol Dehydrogenase from Thermoanaerobacter ethanolicus
}

Musa M. Musa, Karla I. Ziegelmann-Fjeld, Claire Vieille, J. Gregory Zeikus, and Robert S. Phillips ${ }^{*}$

List of Contents

1. Optical rotation for compounds $(S)-\mathbf{1 b}-(S)-\mathbf{5 b}$ and $(S)-\mathbf{7 b}-(S)-\mathbf{9 b}$

2. GC chiral column analysis

3. ${ }^{1} \mathrm{H}$ NMR for compound (S)-1b

4. ${ }^{1} \mathrm{H}$ NMR and ${ }^{13} \mathrm{C}$ NMR for compound $(S)$-2b

5. ${ }^{1} \mathrm{H}$ NMR and ${ }^{13} \mathrm{C}$ NMR for compound (S)-3b

6. ${ }^{1} \mathrm{H}$ NMR and ${ }^{13} \mathrm{C}$ NMR for compound $(S)-4 \mathbf{b}$

7. ${ }^{1} \mathrm{H}$ NMR and ${ }^{13} \mathrm{C}$ NMR for compound $(S)-5 \mathbf{b}$

8. ${ }^{1} \mathrm{H}$ NMR and ${ }^{13} \mathrm{C}$ NMR for compound $(+)-(2 S, 3 S)-\mathbf{6 b}$

9. ${ }^{1} \mathrm{H}$ NMR and ${ }^{13} \mathrm{C}$ NMR for compound (-)-(2S,3S)-6c

10. ${ }^{1} \mathrm{H}$ NMR and ${ }^{13} \mathrm{C}$ NMR for compound $(S)-7 \mathbf{b}$

11. ${ }^{1} \mathrm{H}$ NMR and ${ }^{13} \mathrm{C}$ NMR for compound (S)-8b

12. ${ }^{1} \mathrm{H}$ NMR and ${ }^{13} \mathrm{C}$ NMR for compound (S)-9b

13. Supporting references
Page number

S2, S3

S3,S4

S5

S6

S7

S8

S9

S10

S11

$\mathrm{S} 12$

S13

S14

S15 


\section{Optical rotation for compounds $(S)-1 \mathrm{~b}-(S)-5 \mathrm{~b}$ and $(S)-7 \mathrm{~b}-(S)-9 \mathrm{~b}$ :}

(S)-4-Phenyl-2-butanol ((S)-1b): $[\alpha]^{20}+16.5\left(c 1.81, \mathrm{CHCl}_{3}\right)>99 \%$ ee, lit. ${ }^{1 \mathrm{a}}[\alpha]_{\mathrm{D}}$ +17.4 c $\left.1.80, \mathrm{CHCl}_{3}\right) 99 \%$ ee. Spectral data were consistent with that reported previously. $^{1 \mathrm{~b}}$

(S)-3-Hydroxy-1-phenyl-1-butanone ((S)-2b): $[\alpha]^{20}{ }_{\mathrm{D}}+61.5\left(c 1.65, \mathrm{CHCl}_{3}\right)>99 \%$ ee, lit. $^{2 \mathrm{a}}[\alpha]^{25}+48.5\left(c 2.8, \mathrm{CHCl}_{3}\right) 77 \%$ ee. Spectral data were consistent with that reported previously. $^{2 b}$

(S)-4-Phenyl-3-butene-2-ol ((S)-3b): $[\alpha]^{20}$ - $33.1\left(c\right.$ 1.02, $\left.\mathrm{CHCl}_{3}\right)>99 \%$ ee, $(R)-3 \mathbf{b}$ lit. ${ }^{3 \mathrm{a}}[\alpha]^{20}{ }_{\mathrm{D}}+27.0\left(\mathrm{c} 0.5, \mathrm{CHCl}_{3}\right) 84 \%$ ee. Spectral data were consistent with that reported previously. $^{3 b}$

(S)-4-(4-Methoxyphenyl)-2-butanol ((S)-4b): $\left.[\alpha]^{20}{ }_{\mathrm{D}}+12.8(c) 2.41, \mathrm{CHCl}_{3}\right) 91 \%$ ee, lit. ${ }^{4}[\alpha]^{20}{ }_{\mathrm{D}}+30.9\left(c 1.0, \mathrm{CHCl}_{3}\right) 94 \%$ ee. Spectral data were consistent with that reported previously. 4

(S)-Phenoxy-2-propanol ((S)-5b): $[\alpha]^{20}{ }_{\mathrm{D}}+30.7\left(c\right.$ 1.32, $\left.\mathrm{CHCl}_{3}\right)>99 \%$ ee, lit. $^{5 \mathrm{a}}[\alpha]^{20}{ }_{\mathrm{D}}$ $+28.9\left(\mathrm{c} 1.10, \mathrm{CHCl}_{3}\right) 99 \%$ ee. Spectral data were consistent with that reported previously. $^{5 b}$

(S)-1-Phenyl-2-propanol ((S)-7b): $[\alpha]^{20}{ }_{\mathrm{D}}+14.5\left(c 1.04, \mathrm{CHCl}_{3}\right) 37 \%$ ee, lit. $^{6 \mathrm{a}}[\alpha]^{25}$ $+42.2\left(\mathrm{c} 1.0, \mathrm{CHCl}_{3}\right)>99 \%$ ee. Spectral data were consistent with that reported previously. $^{6 b}$

(S)-4-(4-Methoxyphenyl)-2-propanol ((S)-8b): $[\alpha]^{20}{ }_{\mathrm{D}}+16.3\left(c 1.86, \mathrm{CHCl}_{3}\right)>99 \%$ ee, lit. ${ }^{7}[\alpha]^{20}{ }_{\mathrm{D}}+27.0\left(c 4.40, \mathrm{CHCl}_{3}\right) 95 \%$ ee $)$. Spectral data were consistent with that reported previously. ${ }^{6 a}$ 
(S)-2-Tetralol ((S)-9b): $[\alpha]^{20}-43.77\left(c 0.911, \mathrm{CHCl}_{3}\right) 71 \%$ ee, lit. $^{8 \mathrm{a}}[\alpha]^{20}{ }_{\mathrm{D}}-29.6(c$

$\left.0.50, \mathrm{CHCl}_{3}\right) 85 \%$ ee). Spectral data were consistent with that reported previously. ${ }^{8 \mathrm{~b}}$

\section{GC chiral column analysis}

A supelco $\beta$-Dex 120 chiral column $(30 \mathrm{~m} \times 0.25 \mathrm{~mm}$ [i.d.], $0.25 \mu \mathrm{m}$ film thickness $)$ was used to determine the $\%$ conversion and the ee of the produced alcohols or their corresponding acetates. The acetates were prepared using a method previously reported in literature. ${ }^{3 \mathrm{~b}}$ The injector temperature was $250^{\circ} \mathrm{C}$ and the detector temperature was $300^{\circ} \mathrm{C}$. The flow rate was $19.0 \mathrm{psi}$. The column was programmed between $120^{\circ} \mathrm{C}$ and $170^{\circ} \mathrm{C}$. 


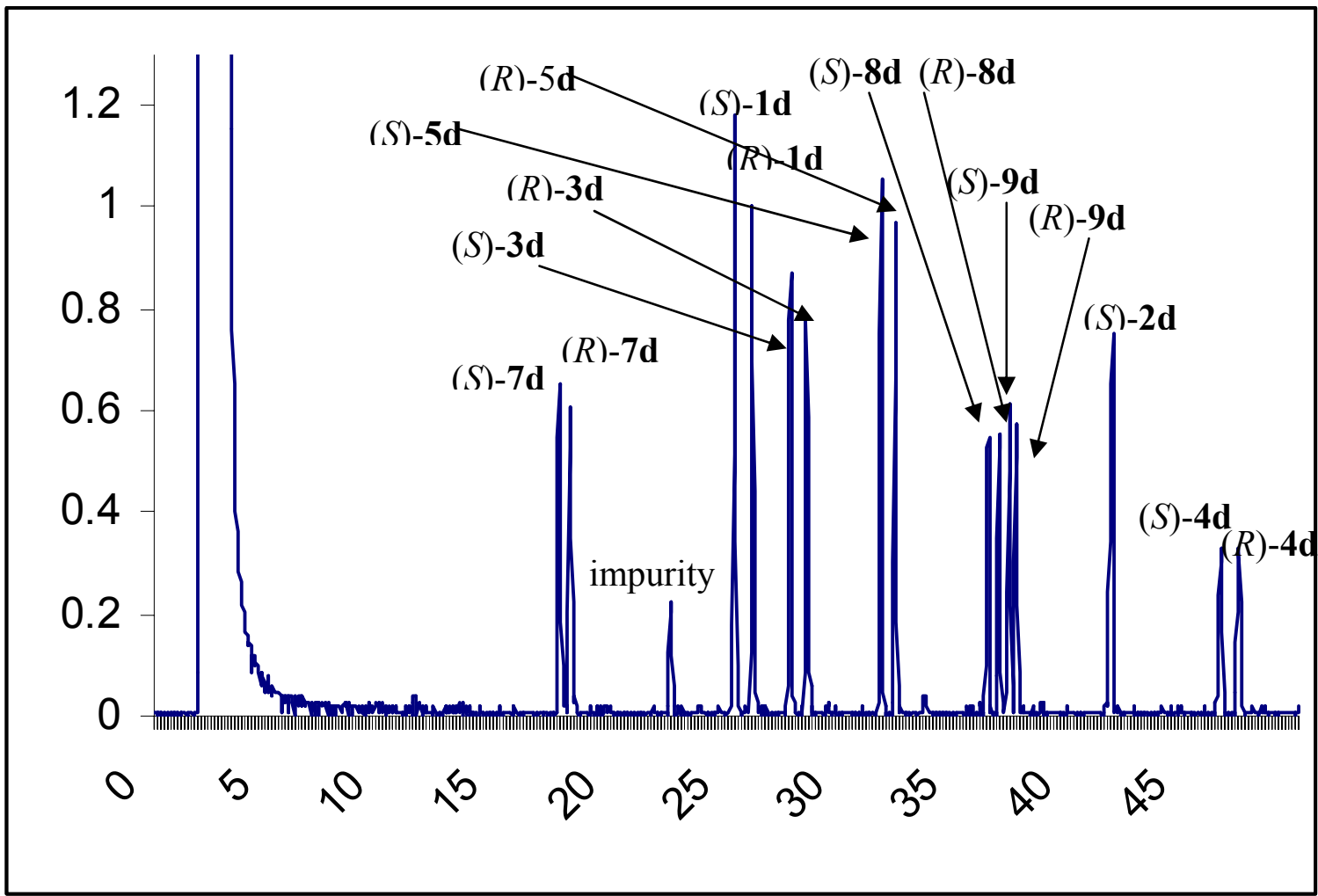

OAC

- OAC

OAC

OAC

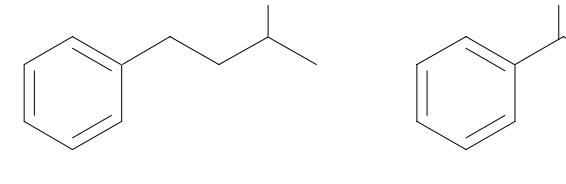

rac-1d

(S)-2d

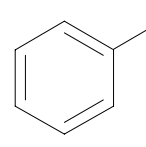

$r a c-3 d$ rac-4d

OAC

0

OAc

$\mathrm{MeO}$

rac-7d

rac-8d

rac-9d

OAc

\section{$\mathrm{MeO}$}


(S)-4-Phenyl-2-butanol ((S)-1b)

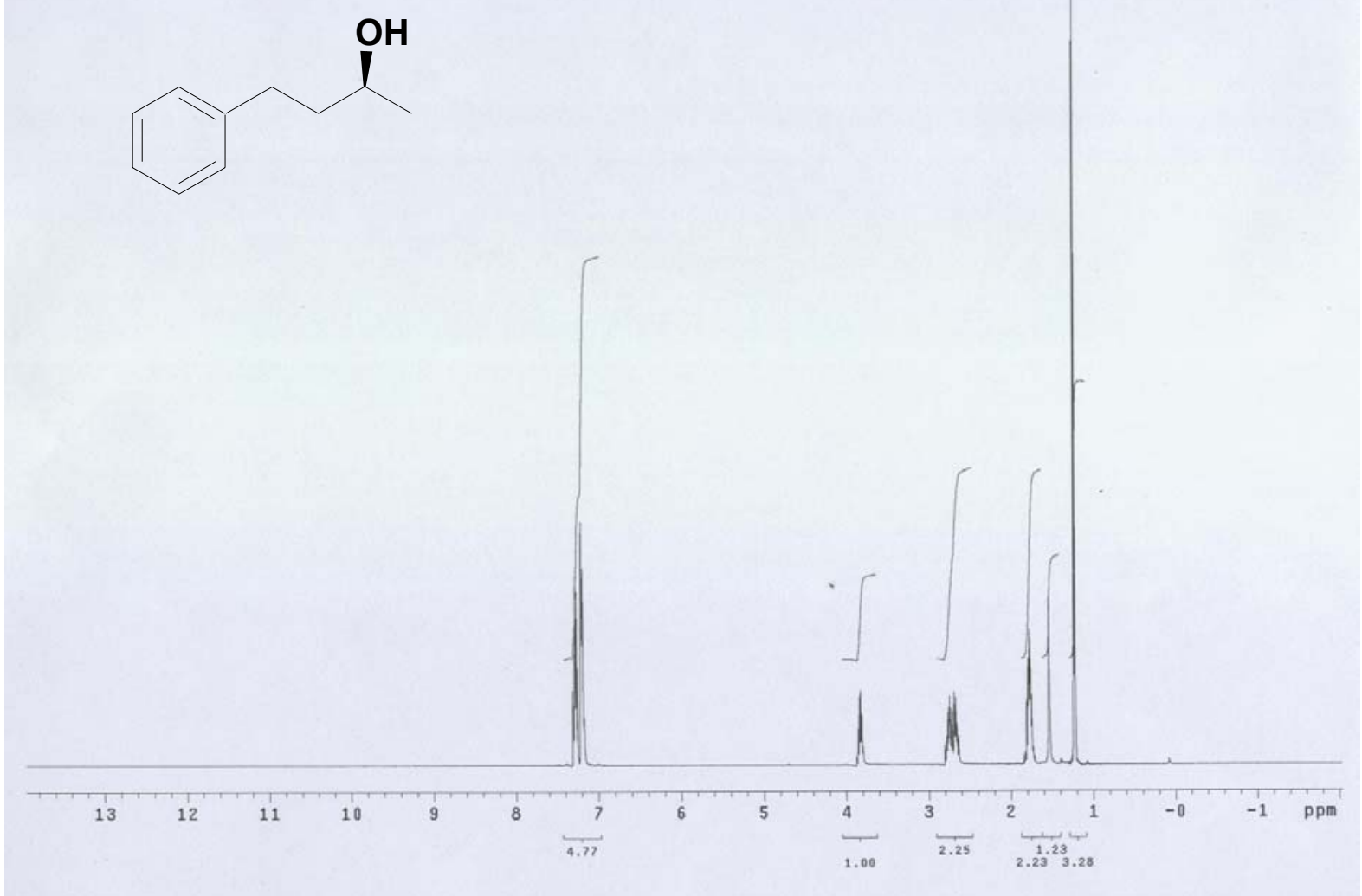


(S)-3-Hydroxy-1-phenyl-1-butanone ((S)-2b):
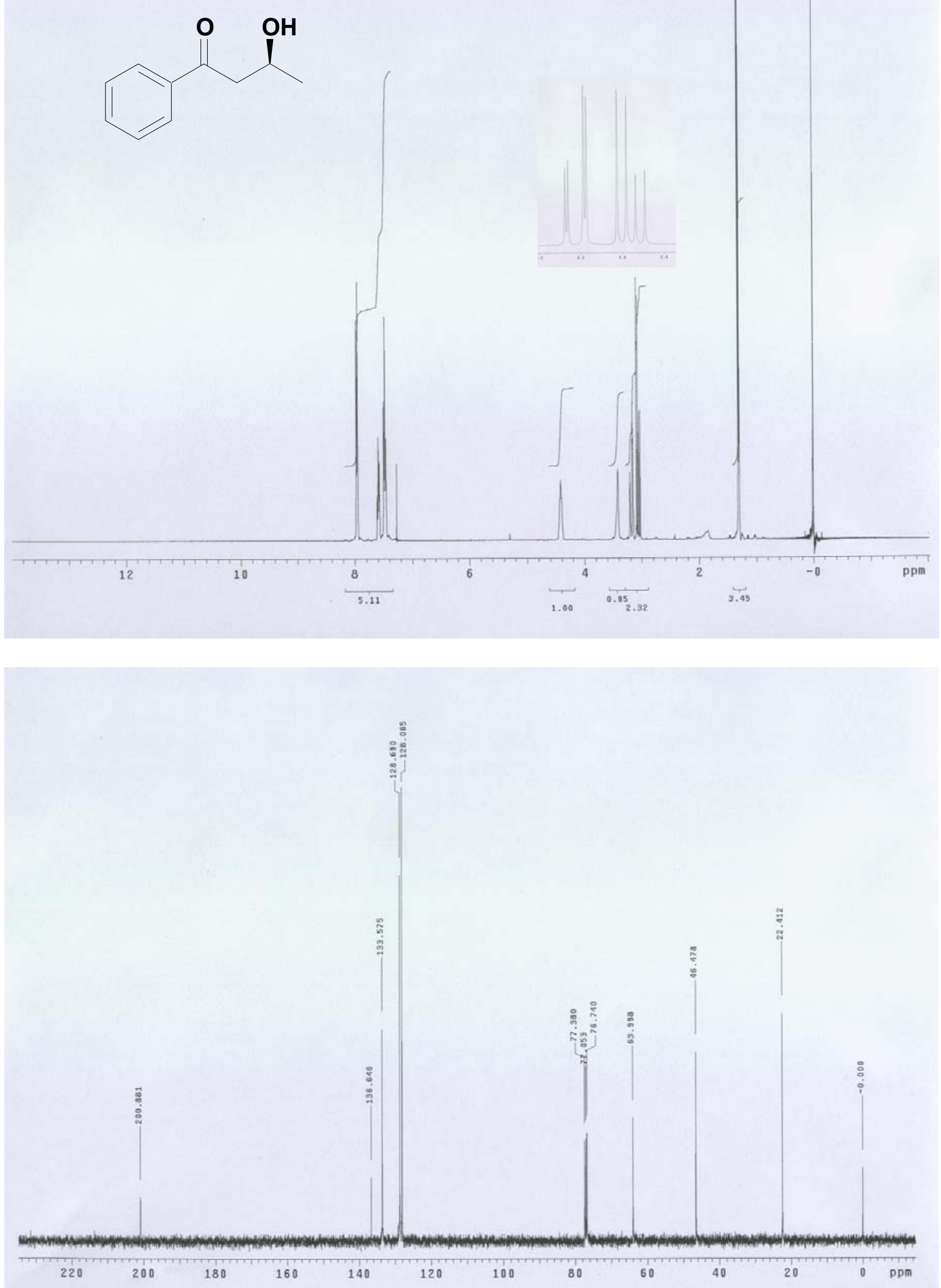
(S)-4-Phenyl-3-butene-2-ol ((S)-3b):

$\mathrm{OH}$
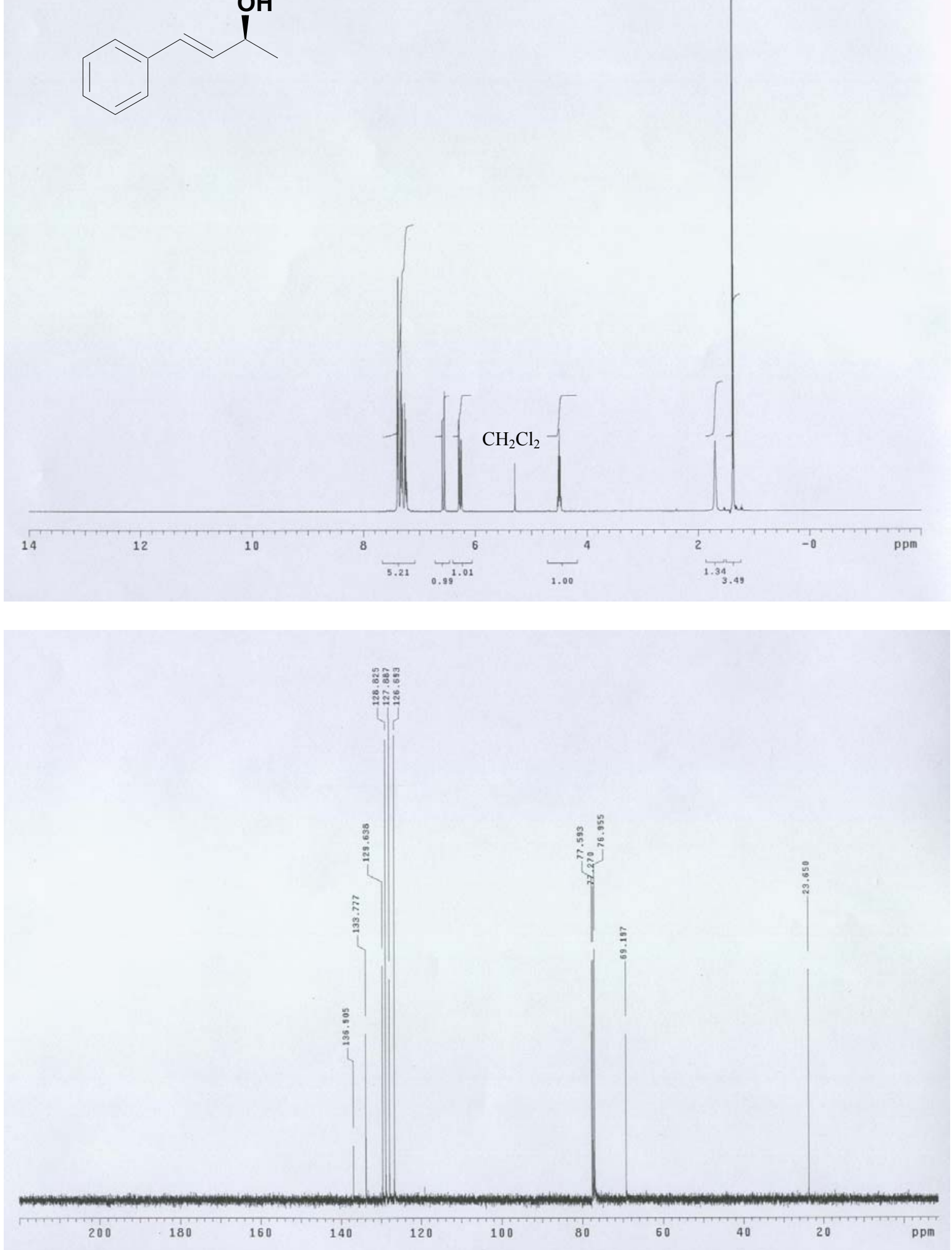
(S)-4-(4-Methoxyphenyl)-2-butanol ((S)-4b):
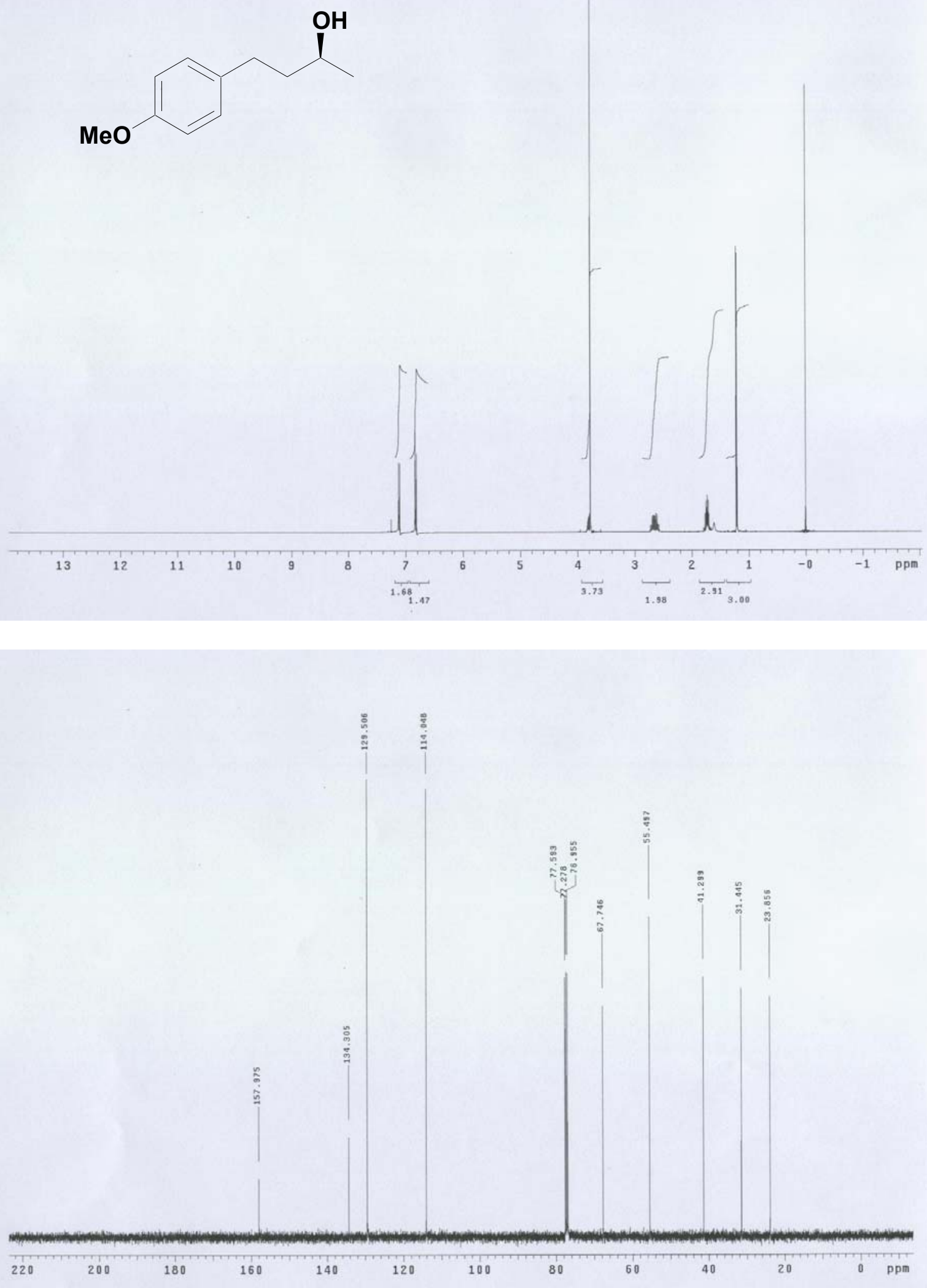
(S)-Phenoxy-2-propanol ((S)-5b):
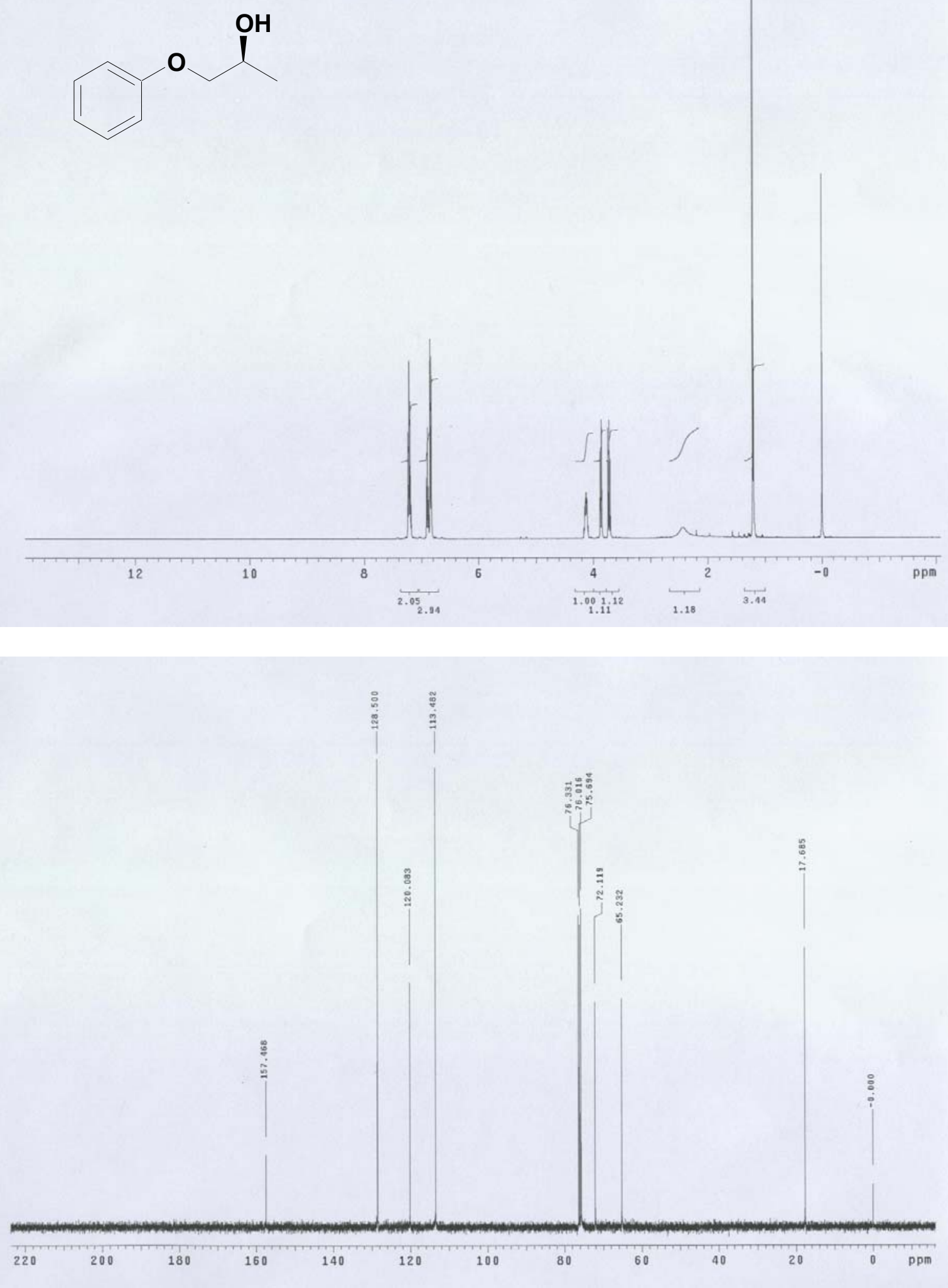
(+)-(2S,3R)-3-Chloro-4-(4-chlorophenyl)-2-butanol ((+)-(2S,3R)-6b):
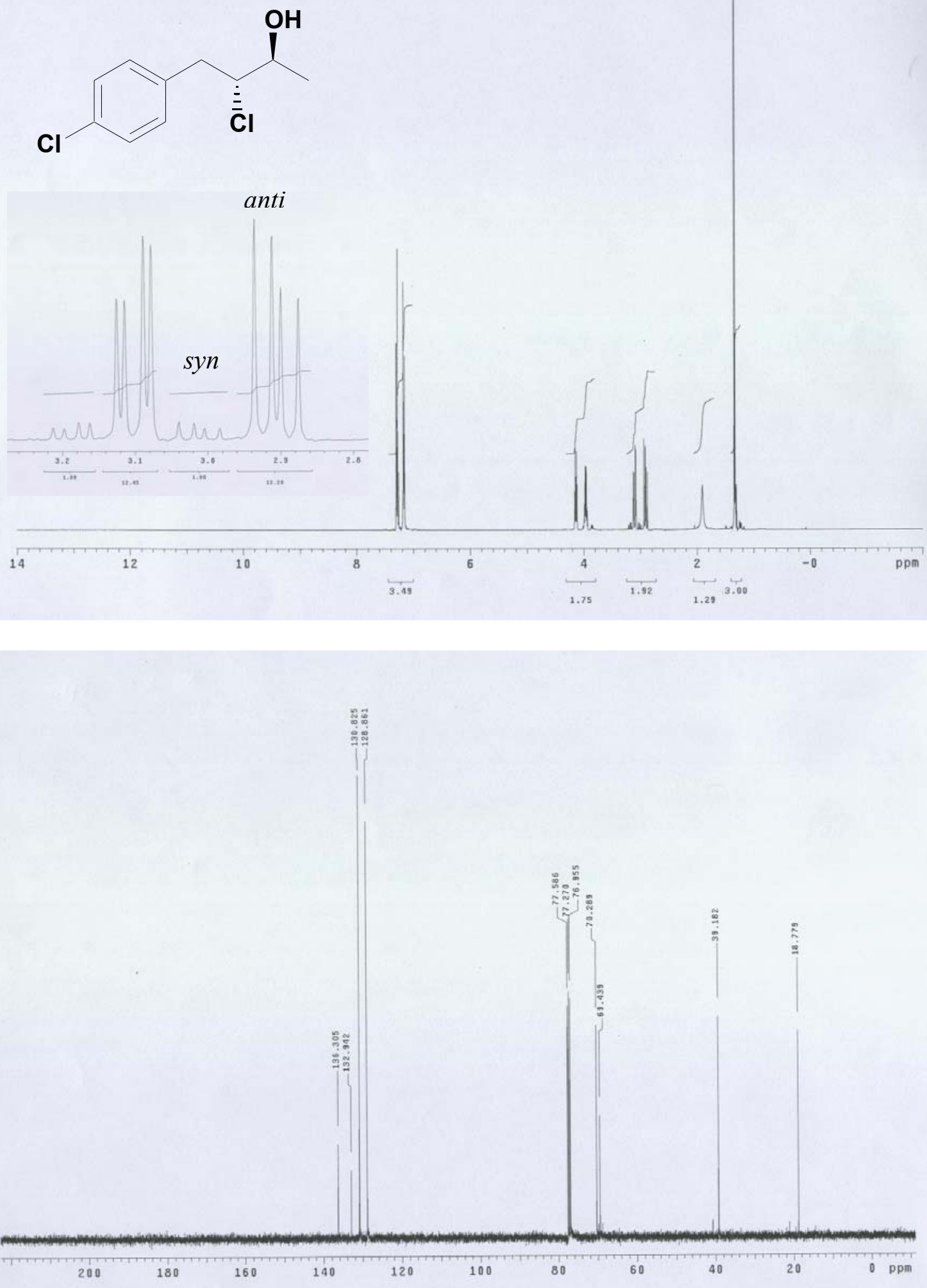
(-)-(2S,3S)-4-(4-Chlorophenyl)-2,3-epoxybutane ((-)-2S,3S)-6c):
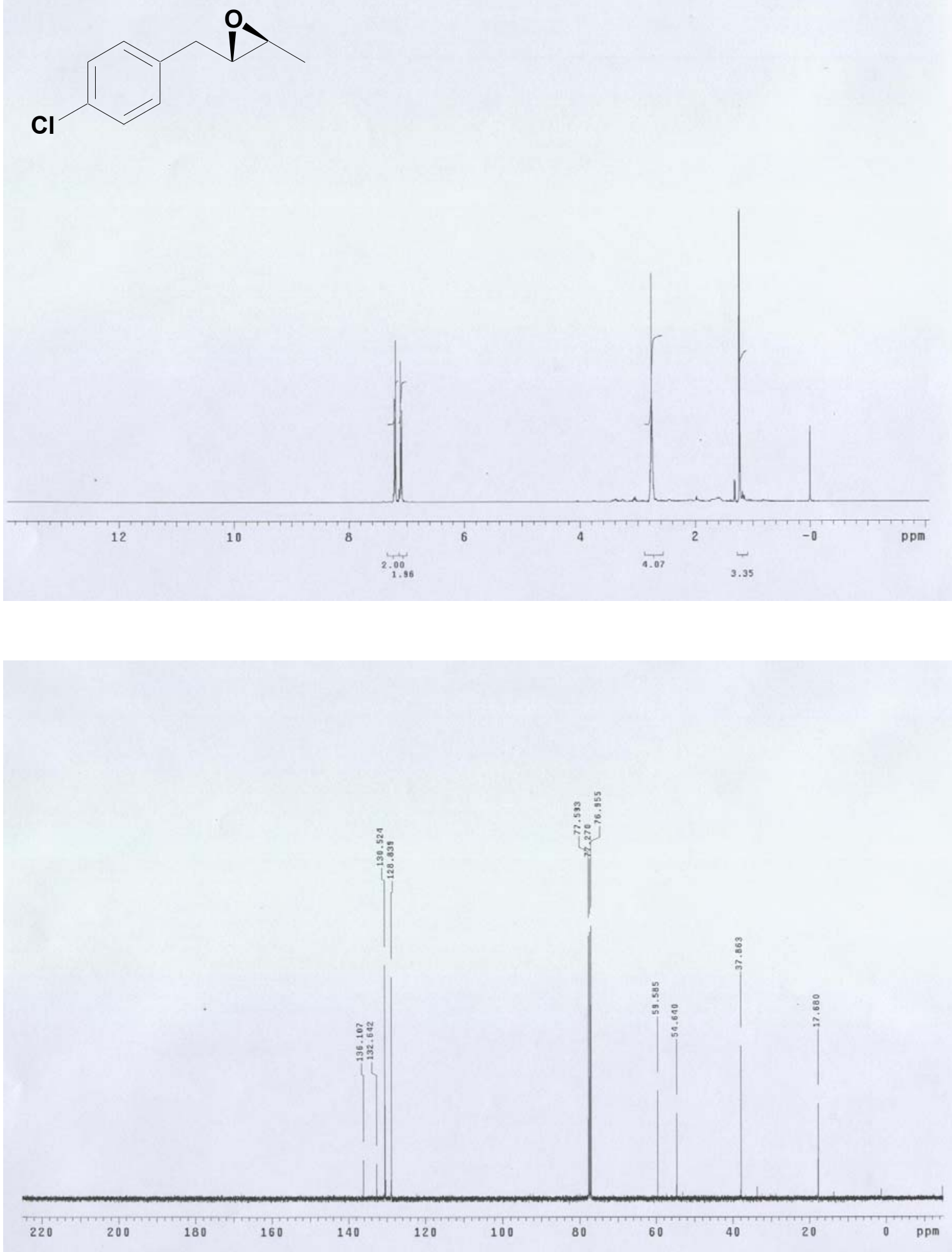
(S)-1-Phenyl-2-propanol ((S)-7b):
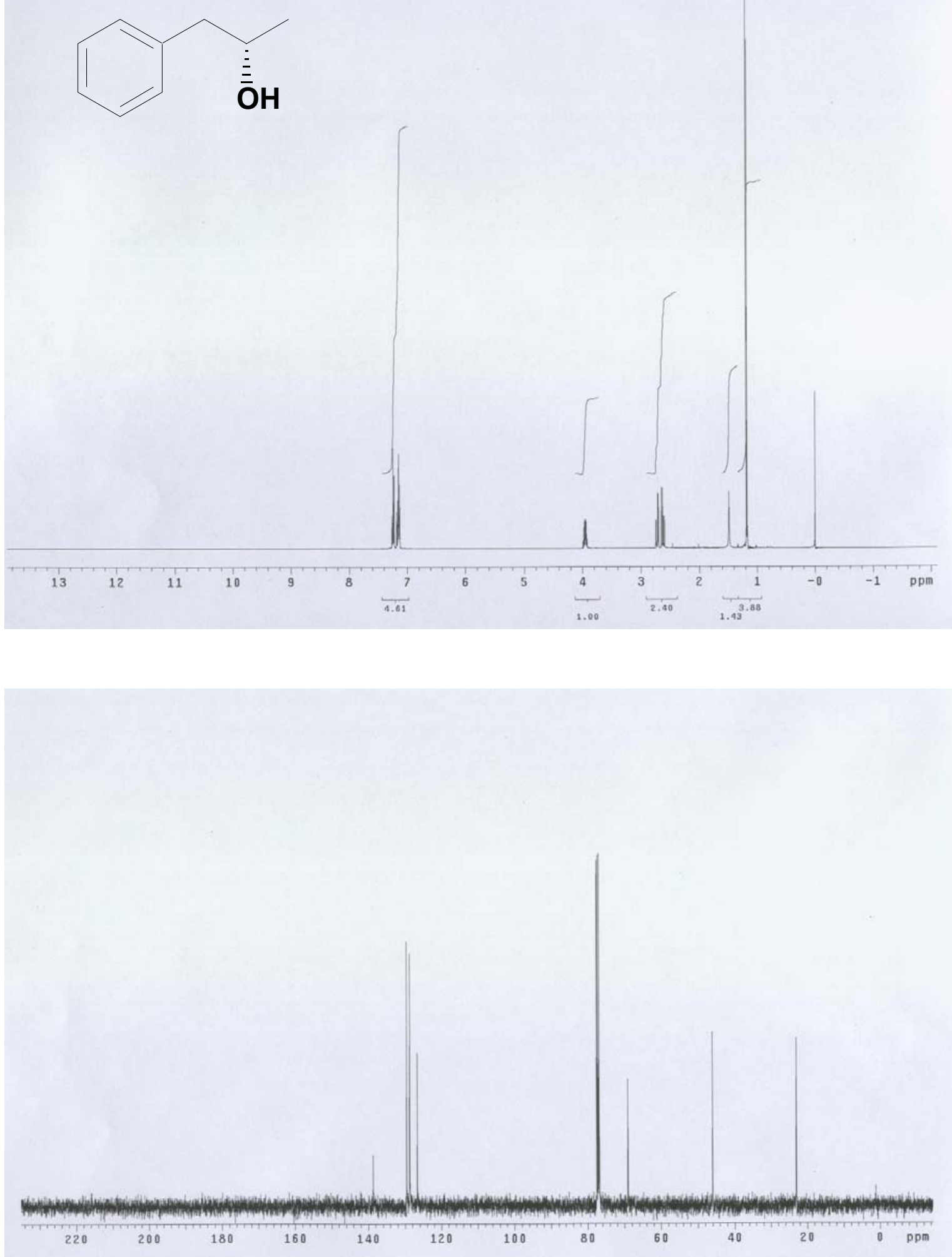
(S)-4-(4-Methoxyphenyl)-2-propanol ((S)-8b):
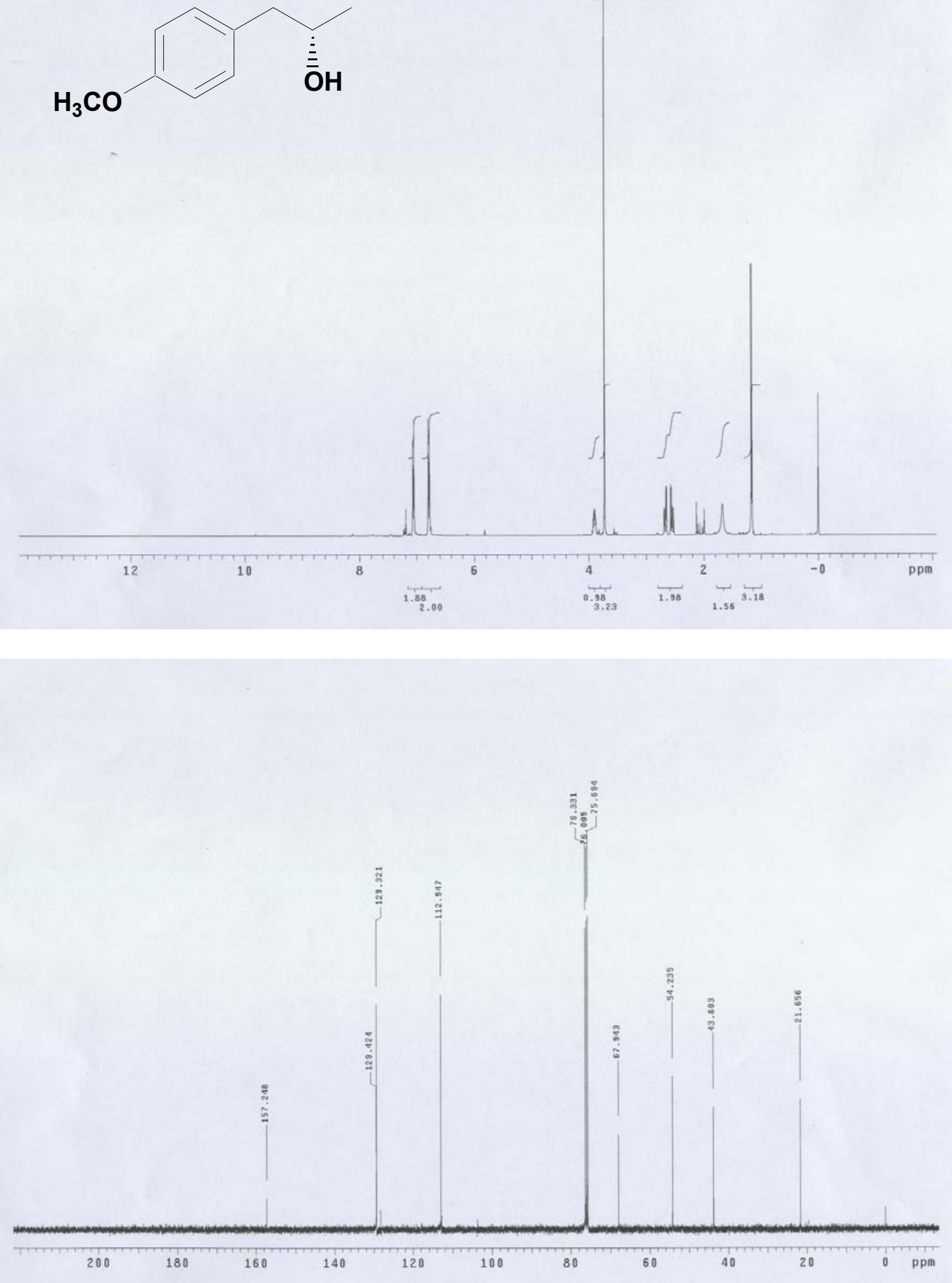

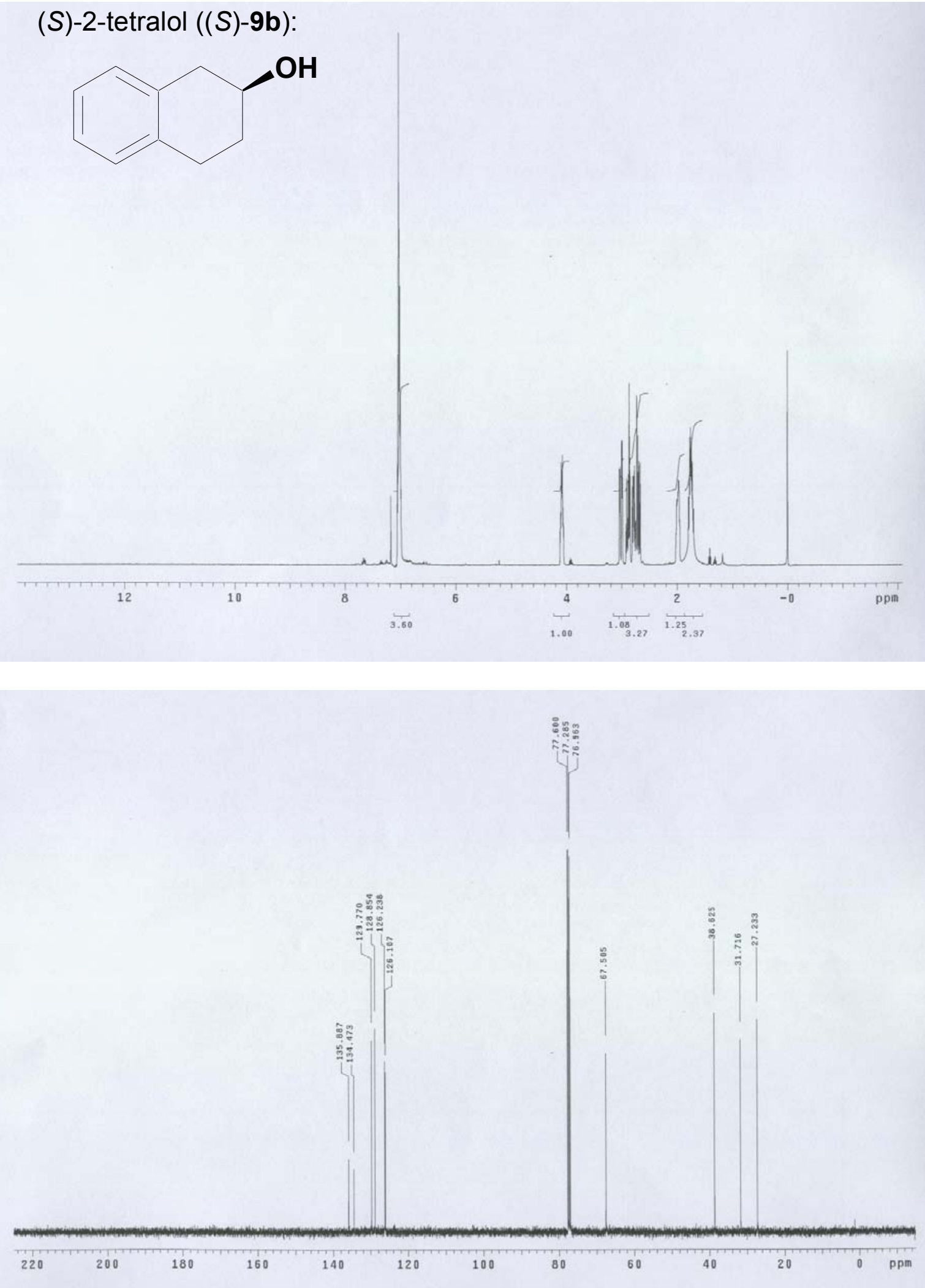


\section{Supporting References:}

1. (a) Nakamura, K.; Inoue, Y.; Matsuda, T.; Misawa, I. J. Chem. Soc. Perkin Trans. 1 1999, 2397-2402. (b) Kuwano, R.; Uemura, T.; Saitoh, M.; Ito, Y. Tetrahedron: Asymmetry 2004, 15, 2263-2271.

2. (a) Ahmad, K.; Koul, S.; Taneja, S. C.; Singh, A. P.; Kapoor, M.; Riyaz-ul-Hassan; Verma, V.; Qazi, G. N. Tetrahedron: Asymmetry 2004, 15, 1685-1692. (b) Kawakami, T.; Shibata, I.; Baba, A. J. Org. Chem. 1996, 61, 82-87.

3. (a) Mastranzo, V. M.; Quintero, L.; Parrodi, C. A.; Juaristi, E.; Walsh, P. J. Tatrahedron 2004, 60, 1781-1789. (b) Ghanem, A.; Schuuring, V. Tetrahedron: Asymmetry 2003, 14, 57-62.

4. Donzelli, F.; Fuganti, C.; Mendozza, M.; Pedrocchi-Fantoni, G.; Servi, S.; Zucchi, G. Tetrahedron: Asymmetry 1996, 7, 3129-3134.

5. (a) Nakamura, K.; Takenaka, K.; Fujii, M.; Ida, Y. Tetrahedron Lett. 2002, 43, 3629-3631. (b) Dragovich, P. S.; Prins, T. J.; Zhou, R. J. Org. Chem. 1995, 60, $4922-4924$.

6. (a) Erdélyi, B.; Szabó, A.; Seres, G.; Birincsik, L.; Ivanics, J.; Szatzker, G.; Poppe, L. Tetrahedron: Asymmetry 2006, 17, 268-274. (b) Ley, S. V.; Mitchell, C.; Pears, D.; Ramarao, C.; Yu, J.; Zhou, W. Org. Lett. 2003, 5, 4665-4668.

7. Ferrabochi, P.; Grisenti, P.; Manzocchi, A.; Santanielo, E. J. Chem. Soc. Perkin Trans. 1 1990, 2469-2474.

8. (a) Stampfer, W.; Kosjek, B.; Faber, K.; Kroutil, W. J. Org. Chem. 2003, 68, 402406. (b) Orsini, F.; Sello, G.; Travaini, E.; Gennaro, P. D. Tetrahedron: Asymmetry 2002, 13, 253-259. 